\title{
Recurrent Incisional Hernia Associated with Interfer- on Treatment for Virus C Hepatitis: Case Report
}

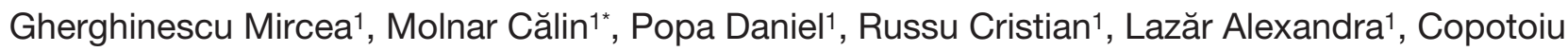 \\ Constantin ${ }^{1}$ \\ 1 University of Medicine and Pharmacy, Tîrgu Mureș, Romania
}

Background: Hepatitis C prevalence in Romania is 3.5\%. Nowadays, the treatment of this condition comprise of interferon. One of the interferon's side effects is the reduction of collagen synthesis, substance that is necessary in the process of abdominal wall healing. Case report: We report the case of a 56 years old female patient, admitted in our Clinic for a giant, recurrent incisional hernia. The patient's history was eventful: a hysterectomy for uterine fibroma in 2009, incisional hernia repair in 2010, the treatment with Interferon in 2011 and 2012 for viral hepatitis C. A well represented subcutaneous tissue is observed intraoperator, a wall defect of $15 \mathrm{~cm}$ in diameter with a $5 \mathrm{~mm}$ thick muscle aponeurosis lay. We performed abdominal wall plasty by components separation technique, reinforced with a polypropylene mesh disposed on lay. Postoperative analgesia was provided by inserting a wound catheter through which Ropivacaine $0,5 \%$ was continuously injected for 72 hours. The postoperative evolution was uneventful, the patient being discharged 7 days after the surgical intervention. Conclusions: The treatment with Interferon of hepatitis $\mathrm{C}$ can favor the recurrence of an incisional hernia. The Oscar Ramirez procedure seemed to be the best choice for surgical treatment of this giant incisional hernia. Postoperative analgesia can be accomplished by a wound catheter through which Ropivacaine $5 \%$ is continuously infiltrated.

Keywords: interferon, incisional hernia, virus $\mathrm{C}$ hepatitis, catheter, pain

Received: 18 August 2015 / Accepted: 03 September 2015

\section{Introduction}

A systematic review of hepatitis $C$ virus epidemiology in Europe shows a prevalence of the disease in Romania, of approximately $3,5 \%$ [1], with variability according with the living environment (urban- $2.76 \%$ or rural- $4.43 \%$ ) [1]. The reported prevalence of HCV infection in healthy adults was $4.5 \%$ as compared with $16.9 \%$ in the high-risk group of orphans [2]. The treatment of this condition is currently done with Interferon. One of its side effects is the reduction of collagen synthesis [3], substance which is needed in the process of healing. Postoperative hernias are a frequent complication of laparotomies, Poelman reported that at least $15 \%$ of the patients, following open abdominal surgery, will develop an incisional hernia in the following 10 years [4]. Giant postoperative hernias are hernias with a parietal defect of over $10 \mathrm{~cm}$ [5]. The cure of these postoperative hernias is a challenge for the surgeon.

\section{Case report}

We present the case of a 56 years old white obese female (Body Mass Index= 32), admitted in our Clinic for elective surgery, with the following diagnose: recurrent sub umbilical, giant, median incisional hernia. The patient's history was eventful: a hysterectomy for uterine fibroma in 2009, incisional hernia repair in 2010, treatment with Interferon in 2011 and 2012 for viral hepatitis C. We want to highlight the fact that at the admittance, the viral markers for viral hepatitis $\mathrm{C}$, were negative. Intraoperator, an $8 \mathrm{~cm}$

* Correspondence to: Călin Molnar

E-mail: molnar.calin@yahoo.com thick subcutaneous tissue is observed, a wall defect of 15 $\mathrm{cm}$ in diameter, a muscle aponeurosis lay poorly represented- $5 \mathrm{~mm}$ thick- with transillumination present (Figure1).

We performed extended adhesiolysis, explored the peritoneal cavity, insisting on the liver, with no appearance of cirrhosis. We performed the abdominal wall plasty by the separation of components, procedure described by Oscar Ramirez and co. in 1990, and we strengthen it with a polypropylene mesh placed on lay.

The Oscar Ramirez procedure consist in:

1. Skin and subcutaneous fat are dissected from the rectus sheath and the external oblique aponeurosis

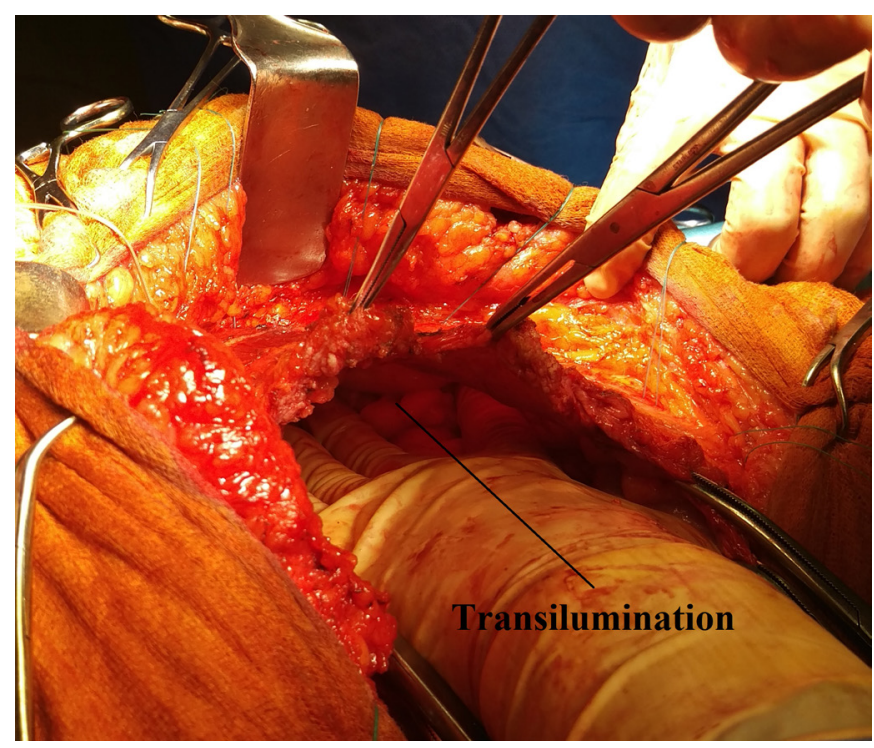

Fig. 1.Intraoperative aspect highlighting the thickness of the abdominal wall 
2. The external oblique aponeurosis is transected longitudinally, about $2 \mathrm{~cm}$ from the lateral margin of the rectus sheath, on each side, unbounding it from the internal oblique's aponeurosis, in an almost avascular plane. The method allows an approach on the median line with about 6 to $10 \mathrm{~cm}$ in the superior abdominal plane, of 14 to $20 \mathrm{~cm}$ in the middle abdominal plane and of 2 to $6 \mathrm{~cm}$ in the inferior abdominal plane, which allows closing of the abdominal wall defect.

3 . Another $2 \mathrm{~cm}$ can be earned by practicing two incisions in the posterior sheath of the rectus abdominis, with the division and suture of these two aponeurotic flaps, on the median line.

Whereas the procedure needs extensive parietal division, blamed for intense pain, the postoperative analgesia was provided by placing a wound catheter above the polypropylene mesh (figure2). Ropivacaine $0,5 \%$ was continuously injected through this catheter. The initial infusion rate was $10 \mathrm{ml} /$ hour, gradually decreased to $5 \mathrm{ml} /$ hour. The dosage was adjusted to the patient's needs as evaluated by VAS (visual analogue scale) to a value of 1-2. The postoperative evolution was uneventful, the patient being discharged 7 days after the surgical intervention

The patient was included, under a written inform consent, in a clinical study about local analgesia in the postoperative care after incisional hernia treatment. The study was conducted with the approval of the Hospital and University Ethics Committee.

\section{Discussions}

The appearance and recurrence of the incisional hernias is influenced besides obesity, by postoperative parietal suppurations, postoperative physical effort, technical deficiencies of surgery and disorders of collagen metabolism [6-8]. The quantitative and qualitative alterations of the interstitial collagen are accountable for the deficient healing on the suture site [7].

Although the treatment with Interferon was interrupted for more than 2 years, the resistance structure of the abdominal wall, represented by the muscle- aponeurotic sheath, was only $5 \mathrm{~mm}$ thick which allowed transillumination by the scialytic lamp.

The surgical procedure is a controversial procedure according to some authors, because it has a high rate of cutaneous necrosis $(9.3 \%)$, of seromas $(12.0 \%)$ or postoperative hematoma $(6.7 \%)$ including an unacceptable high recurrence rate(38.7\%) [9]. Other authors, such as T. S. de VriesReilingh, are arguing in a study published in May 2007 in World Journal of Surgery, that there are no statistical significant differences of the above complications on the patients with "Components Separation Technique" and Prosthetic plastyies [10].

In order to enhance the resistance of abdominal wall, we decided to use additionally a polypropylene mesh, which was placed on lay, decreasing the risk of recurrence [11].

Kenneth C. Shestak measures the contractility of the abdominal musculature using the Cybex TEF machine and obtains, postoperatively, a value of $40 \%$ compared to the value from the preoperative period [12]. This demonstrates the superiority of the procedure compared to the ones with substitution mesh, where the contractility on the parietal defect is 0 .

It is a well-known fact that optimizing the postoperative analgesia improves the results and increases the patient's satisfaction [13].

The use of Ropivacaine for postoperative analgesia, through the wound catheter inserted intraoperatively,

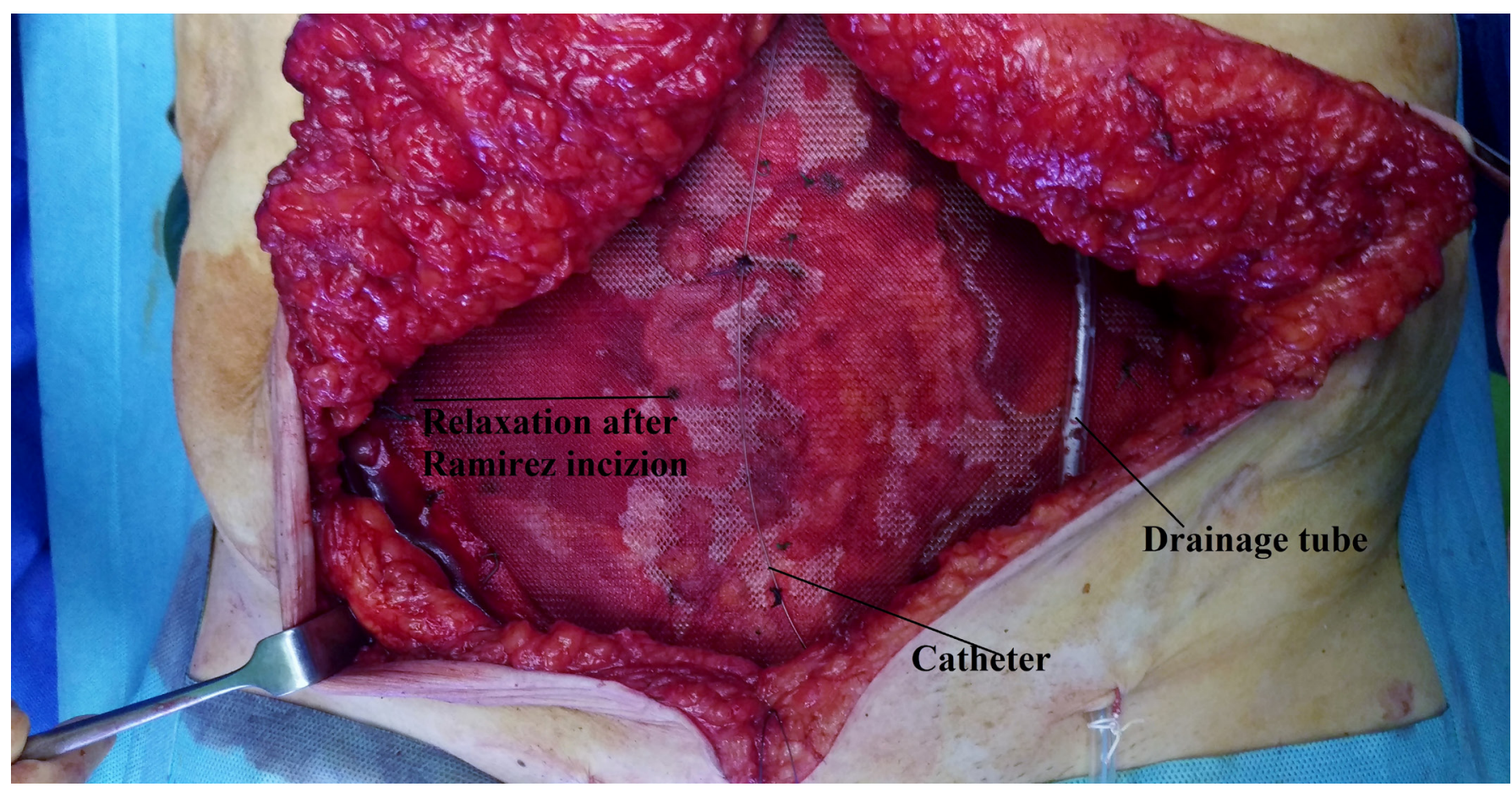

Fig. 2. Intraoperative aspects 
precluded the need for analgesics and non-steroidal antiinflammatory drugs which may harm the liver.

\section{Conclusions}

We submit to the opinions that, although there is not consistently enough evidence to support this, the treatment with Interferon for hepatitis $\mathrm{C}$ can favor the appearance and recurrence of a postoperative incisional hernia.

The Oscar Ramirez procedure, also known by the name of "components separation technique", although controversial, seemed to be the best choice for surgical treatment of this giant incisional hernia, being preferred to substitution mesh plasty.

Continuous wound local analgesia by an supra aponeurotic catheter with Ropivacaine 5\% contributed to the recovery

\section{Acknowlegement}

This paper was published under the framework of European Social Found, Human Resources Development Operational Programme 2007-2013, project no. POSDRU/159/1.5/S/ 136893

\section{Conflict of interest}

None to declare.

\section{References}

1. Cornberg M, Razavi H, Alberti A, et al. A systematic review of hepatitis C virus epidemiology in Europe, Canada and Israel, Liver International. $2011 ; 30-6$
2. Gheorghe L, lacob S, Csiki I. Prevalence of hepatitis C in Romania: Different from European rates? Letters to the Editor Journal of Hepatology. 2008;49:658-663.

3. Jimenez S.A, Freundlich B, Rosenbloom J. Selective Inhibition of Human Diploid Fibroblast Collagen Synthesis by Interferons J Clin Invest. 1984;74:1112-1116.

4. Poelman M, ApersJ, Van den Brand $\mathrm{H}$,et al. The INCH-Trial: a multicentre randomized controlled trial comparing the efficacy of conventional open surgery and laparoscopic surgery for incisional hernia repair. BMC Surgery. 2013;13:18.

5. Muysoms F, Miserez M, Berrevoet F, et al. Classification of primary and incisional abdominal wall herniasHernia. 2009;13:407-414.

6. Rosch R1, Junge K, Knops M, et al. Analysis of collagen-interacting proteins in patients with incisional herniasLangenbecks Arch Surg. 2003;387(11-12):427-32.

7. Klinge $U$, Binnebosel $M$, Mertens PR. Are collagens the culprits in the development of Incisional and inguinal hernia disease? Hernia. 2006;10:472-477.

8. Rosch R, Binnebösel M, Junge $\mathrm{K}$, et al. Analysis of c-myc, PAl-1 and UPAR in patients with incisional hernias Hernia. 2008;12:285-288

9. Slater N, Van Goor H, Bleichrodt R. Large and complex ventral hernia repair using "Components separation technique" without mesh results in a high recurrence rate. J.amjsurg. 2015;209:170-179.

10. De Vries Reilingh TS, Van Goor H, Charbon JA, et al. Repair of Giant Midline Abdominal Wall Hernias:"Components Separation Technique" versus Prosthetic Repair Interim Analysis of a Randomized Controlled Trial World J Surg. 2007;31:756-763.

11 Roth JS, Diaz D, Plymale M, et al. Abdominal Wall Reconstruction Utilizing the Component Separation Technique: Does Reinforcing Mesh Reduce Recurrences? Surgery Curr Res. 2013;3:129.

12. Shestak K, Edington $H$, Johnson R. The Separation of Anatomic Components technique for the reconstruction of massive midline abdominal wall defects: anatomy, surgical technique, applications, and limitations revisited. Plast. Reconstr Surg. 2000;105:731-738.

13. Thornton P, Buggy D. Local anaesthetic wound infusion for acute postoperative pain: a viable option? Br J Anaesth. 2011;107(5):656-658. 